\title{
An Obstacle Detection and Guidance System for Mobility of Visually Impaired in Unfamiliar Indoor Environments
}

\author{
Monther M. Al-Shehabi, Mustahsan Mir, Abdullah M. Ali, and Ahmed M. Ali
}

\begin{abstract}
This paper describes the development of a wearable navigation aid for blind and visually impaired persons to facilitate their movement in unfamiliar indoor environments. It comprises of a Kinect unit, a Tablet PC, a microcontroller, IMU sensors, and vibration actuators. It minimizes reliance on audio instructions for avoiding obstacles and instead guides the blind through gentle vibrations produced in a wearable belt and a light helmet. By differentiating obstacles from the floor, it can detect even relatively small-size obstacles. It can also guide the blind to reach a desired destination (office/room/elevator) within an unfamiliar building with the help of 2-D printed codes, RGB camera of Kinect unit, a compass sensor for orienting the user towards the next direction of movement, and synthesized audio instructions. The developed navigation system has been successfully tested by both blind-folded and blind persons.
\end{abstract}

Index Terms-Electronic mobility aid, navigation assistance system, obstacle detection for visually impaired.

\section{INTRODUCTION}

Millions of people around the world are either blind or visually impaired. A variety of portable or wearable navigation aids have been developed to assist them in their movement in indoor and outdoor environments. An extension of the age-old white cane is a virtual white cane that a blind person can swing around to sense the environment, measure distance to obstacles, and detect surface discontinuities [1]. A wireless ranging system using ultrasonic sensor interfaced to a microcontroller with Bluetooth transceiver and an application running on a smart phone has been developed to detect the presence of obstacles with high accuracy [2]. Two computerised devices, NavBelt and GuideCane, based on advanced robotics obstacle-avoidance technologies are presented in [3]. Navbelt utilizes a belt equipped with an array of ultrasonic sensors. It provides acoustic signals via a set of stereo earphones to guide the user around obstacles. However, for fast-walking users, it becomes difficult to comprehend the guidance signals. This problem was solved by modifying NavBelt to develop a GuideCane. This solution required a wheeled device pushed ahead of the user via an attached cane. A device that allows 3-D space perception by sonification of range information obtained via a laser range sensor is described in [4]. This device can detect corners and depth discontinuities and provides a perception of surrounding space. A navigation assistance system for visually impaired

Manuscript received January 9, 2014; revised April 9, 2014.

M. M. Al-Shehabi was with the Department of Electrical Engineering at Ajman University of Science and Technology, Ajman, UAE.

M. Mir, Abdullah M. Ali, and Ahmed M. Ali are with Ajman University of Science and Technology, Ajman, UAE (e-mail: m.mir@ ajman.ac.ae). was developed using a vision-sensor mounted headgear and stereo earphones [5]. The captured image was processed by using fuzzy clustering algorithms for identification of obstacles.

A portable auditory guide system using a mini-CCD camera, a DSP unit, and an earphone is presented in [6]. In this system, the images taken by the camera are processed to detect objects tagged with barcodes. In [7], embedded RFID tags, with stored location information, are utilized for providing indoor navigation for blind or visually impaired. The navigation device communicates with a routing server using GPRS networks to determine the shortest route from the user's current location to the desired destination. An infrastructure-free indoor navigation system for blind with high positioning accuracy is presented in [8]. A navigation system based on wireless mesh network to provide location and orientation information uses proximity sensors for detection of obstacles [9]. For optimal path planning, a server communicates wirelessly with a portable mobile unit.

Using a vision sensor and a remote server for processing the captured image, an assistance device for visually impaired provides acoustic signals to the user for obstacle avoidance [10]. The remote server also determines optimal path and gives directions to the user on the basis of a pre-stored environment map. For smaller (foot-level) obstacles a guide cane is used. The room-to-room navigation system presented in [11] uses two types of RFID tags placed on each door. The navi tags contain navigation information and audio tags contain voice messages. For avoiding obstacles, the blind would rely on a white cane and messages stored in audio tags. Using an augmented white cane with various embedded infrared lights, two infrared cameras, a computer and a smart phone, an indoor navigation system locates obstacles on the route and offers navigation information when the user presses a button on the cane [12]. Many other navigation systems and devices have been reported in the literature. For a survey of portable/wearable obstacle detection/avoidance electronic aids for blind persons, refer to [13].

Since the hearing sense is quite important for blind and they depend a lot on it, the navigation system presented in this paper intentionally does not rely on using audio instructions to avoid obstacles. Instead, a combination of five vibrators is used to guide the movement of blind avoiding various obstacles along the route. Four of these vibrators are on a wearable belt and the fifth one is on a light helmet. The audio instructions are occasionally used to alert the blind about some imminent danger or for providing some useful information other than continual instructions for obstacle avoidance. The obstacle detection technique used in this system distinguishes obstacles from the floor and thereby 
even relatively small-size obstacles can also be detected.

In addition to obstacle detection and avoidance features, the presented system guides the movement of blind towards a desired destination. For this purpose, 2-D printed codes in conjunction with a database developed for the built-environment were used to determine the route for the blind towards the desired destination (office/room, etc). A compass sensor has been utilized to inform the blind, through a speech synthesizer, about his current orientation and to help him adjust the orientation for moving towards the desired destination.

\section{SYSTEM BLOCK DIAGRAM}

The block diagram of complete system is shown in Fig. 1. It uses a Kinect unit developed by Microsoft for the Xbox video game and Windows PCs. It is a motion sensing input device consisting of an IR depth sensor and RGB camera. The IR depth sensor is used to obtain the depth image data of the environment [14]. It captures greyscale image of everything visible in the field of view of the depth sensor. Since the Kinect uses Infrared (IR), it can see through glass. Therefore, it cannot be used reliably for detecting obstacles such as glass doors. Also, since it uses IR, the Kinect will not work in direct sunlight, e.g. outdoors. To overcome the first limitation, ultrasonic sensors were integrated with the developed system for detecting glass doors. As for the second limitation of Kinect, it had no impact on this system as it was developed only for indoor environments.

A Tablet PC is used to process the image data obtained from the Kinect unit. A microcontroller (Atmel XMEGA-A1 Xplained) with accelerometer, gyroscope, and compass sensors interacts with the Tablet PC for detecting the obstacles and alerting the user to avoid these obstacles through a set of five vibrators. For navigation instructions towards a desired destination, a number of 2-D printed codes are placed on different corners inside the indoor environment to cover the desired area. Each code has a unique ID linked to a specific instruction text. The 2-D code image acquired by the Kinect unit and processed by the Tablet PC is utilized to provide audio instructions to the user.

For processing the input data obtained from the actual environment, C\# programming language was used. In addition, AR-toolkit software was used with the RGB camera of the Kinect sensor for detecting the 2-D printed code.

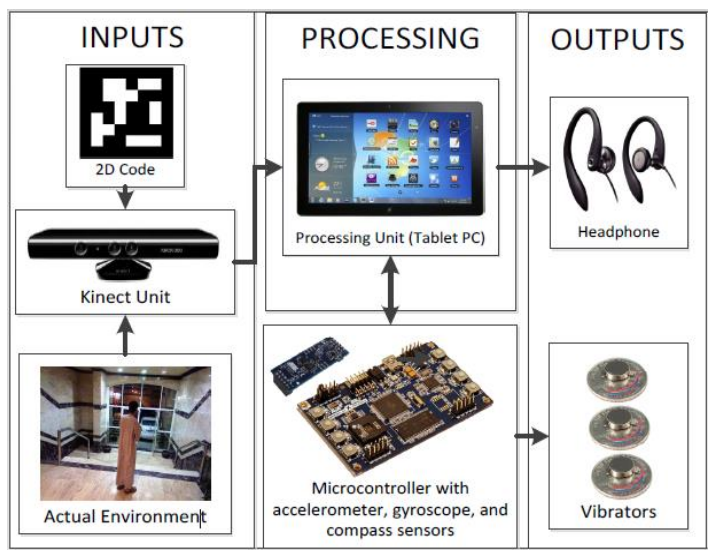

Fig. 1. Block diagram of developed system.

\section{OBStACLE DETECTION}

The obstacle detection process is explained with the help of Fig. 2. The IR depth sensor obtains the depth image data of the actual environment which is sent to the processing unit (Tablet PC). In order to overcome the problem of considering the floor as an obstacle, an algorithm was developed with the use of gyroscope and accelerometer sensors to differentiate between the floor and the obstacles. As a result, relatively small-size obstacles can also be detected. Another algorithm was developed to perform the following sequence of steps. First, it divides the image of depth data into five segments. Each segment has a different depth data according to the depth of the obstacle. The minimum depth of each segment will be considered and the rest will be ignored in order to detect the closest obstacle. A threshold distance is assigned for each segment to decide when the vibrator(s) will be turned on or off. The belt vibrators are placed such that their vibrations indicate the direction of the obstacle(s). The helmet vibrator changes its vibrating frequency (from 1-4 Hz) to let the blind predict the distance of the obstacle in front of him. Depending upon the direction of the obstacle, the corresponding vibrator(s) on the belt will start vibrating if the distance of the obstacle is within one meter range. The vibrator placed on the user helmet will start vibrating with very low frequency $(1 \mathrm{~Hz})$ if the distance of the obstacle is in the range of 3-4 meters. The vibrating frequency will increase in steps as the obstacle becomes nearer, and can reach up to 4 $\mathrm{Hz}$ when the obstacle is at a distance between 1-2 meters. When the distance of the obstacle becomes less than 1 meter, the vibrator will start vibrating continually. As such, the vibration frequency will indicate to the blind the distance of the obstacle.

Some other systems [15], [16] have also utilized the Kinect sensor for indoor navigation of blind people along with vibrators for alerting the user to avoid obstacles along the user's path. However, by utilizing IMU sensors and by developing an algorithm to differentiate between the floor and the obstacles on the basis of IMU sensors' input, relatively small-size obstacles can also be detected in the presented system. Furthermore, the segmentation algorithm is able to compensate for the tilt of Kinect unit within reasonable range and reasonable rotation speed. The tilt angle was introduced to account for the changes in the user's head orientation.

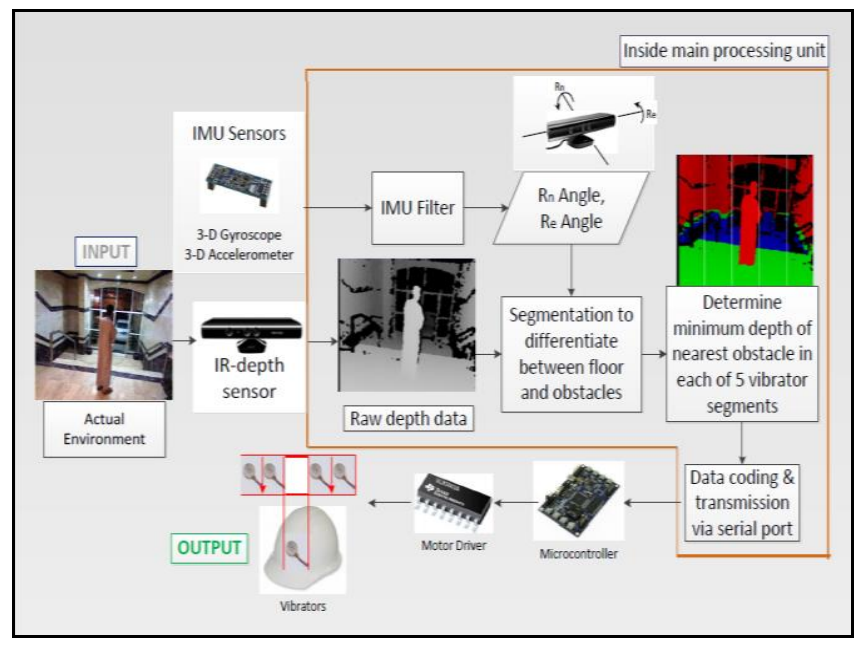

Fig. 2. Block diagram for obstacle detection process. 
The rotation angles of the Kinect unit are explained with the help of Fig. 3 as follows:

Re: Rotation angle around $\mathrm{x}$-axis as in Kinect standards or it is the rotation angle around the axis crossing user's ears. It is negative if the head turns down.

$\mathrm{Rn}$ : Rotation angle around $\mathrm{z}$-axis as in Kinect standards or it is the rotation angle around the axis of user's nose. It is negative if the head turns left. The rotation angles, Re and Rn, are predicted by using the data obtained from IMU sensors (accelerometer and gyroscope).

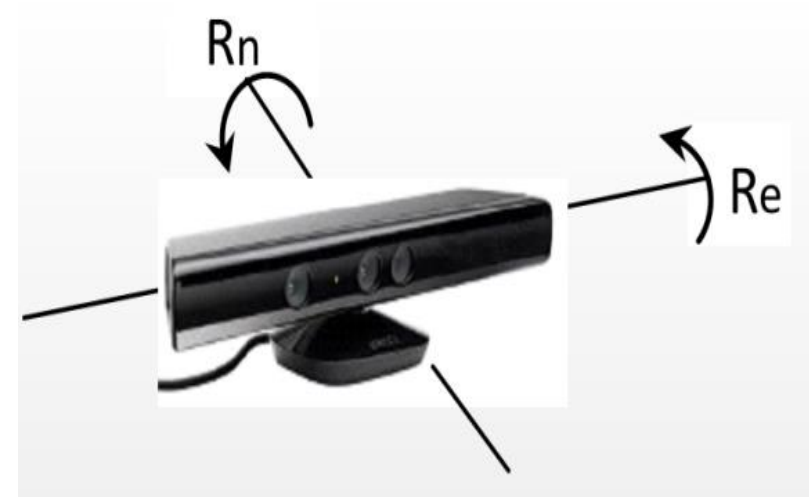

Fig. 3. Rotation angles of Kinect unit.

The algorithm developed for differentiating between the floor and the obstacles was able to differentiate an obstacle from floor with some margin of error. By comparing the depth data value for an object with the predicted floor depth value (within error margin), it is determined, as depicted in Fig. 4, if the object is an obstacle (red pixels) or floor (green pixels).

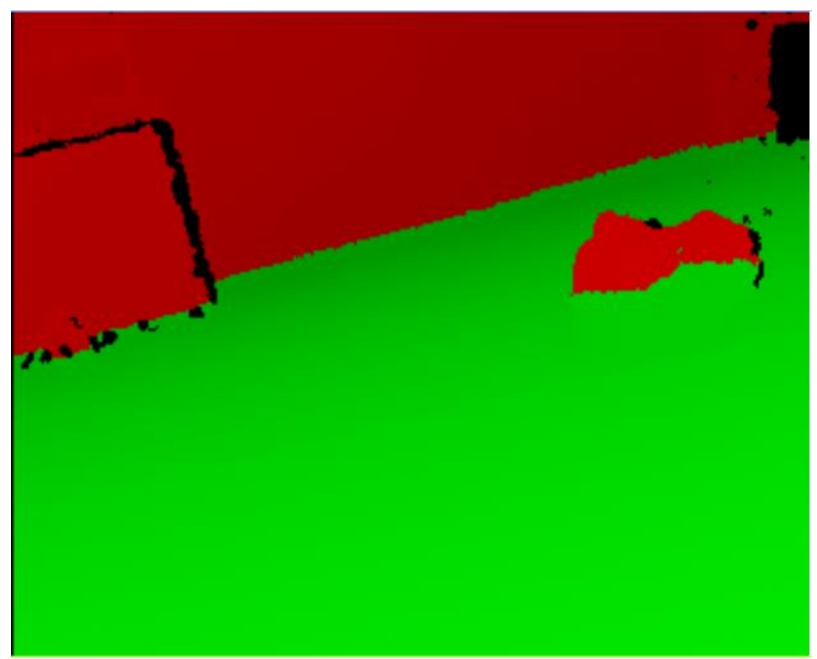

Fig. 4. Red pixels represent wall and obstacle while green pixels represent the floor.

Actual testing of the system showed that the margin of error increased for farther distance and when there was an error in predicting Re due to acceleration of the IMU sensor resulting from user's motion. This error limits the range of obstacles detection which is currently four meters. To reduce the margin of error, the precision of predicting $\mathrm{Re}$ angle should be increased when there is noticeable acceleration in the movement of the user. Also, obstacles appearing suddenly at very short distance (less than $0.4 \mathrm{~m}$ ) were difficult to identify. The same applies to glass obstacles. For these two cases, ultra-sonic sensors were integrated to the detection system.

For alerting the user about the detected obstacle, Atmel XMEGA-A1 Xplained evaluation kit along with Atmel ATxmega128A1 microcontroller and a separate Inertial Two Sensor Board was interfaced to the processing unit (Tablet PC). The XMEGA-A1 Xplained receives data from the Tablet PC and transmits it to vibrators' drivers through UART. Also, the gyroscope, accelerometer, and compass sensors data is transmitted from XMEGA-A1 Xplained to the Tablet PC through UART. The drivers were needed since the vibrators require high power $(3 \mathrm{~V} @ 65 \mathrm{~mA}$ ) that cannot be provided by XMEGA-A1 Xplained. The driver IC (ULN2003A) is a Darlington transistor array. A detailed block diagram of the system developed for providing vibrations in the wearable belt and helmet is shown in Fig. 5 .

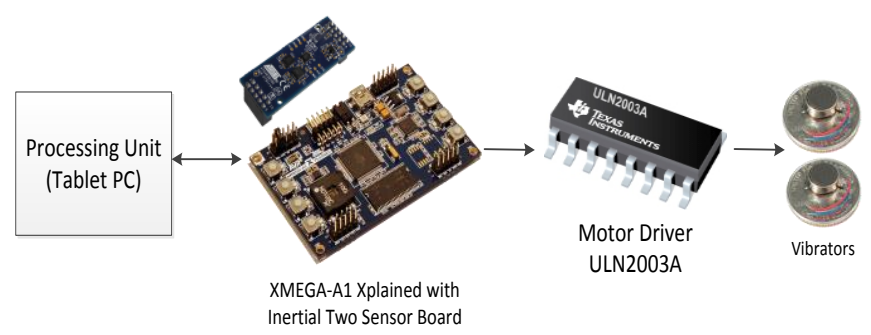

Fig. 5. Vibration system configuration.

\section{NAVIGATION SYSTEM}

The navigation system developed in this work assists the user to reach a specified destination within a building that has a number of 2-D printed codes placed for all destination offices/rooms as well as along the corridors and near the elevators. For the purpose of explanation, the navigation system can be divided into two parts. The block diagram of the first part is shown in Fig. 6. The RGB camera of the Kinect sensor will detect the 2-D printed code using AR-toolkit software. Each code has a unique ID linked to a specific instruction text in the instruction database. This text will be outputted as an audio instruction using a speech synthesizer. The instruction will provide users the required information about their current position and the next direction to be taken for moving towards their desired destination. The maximum distance for detecting the code is up to 4 meters. A 3-D sound beep is given whenever a 2-D code is detected to inform the users about the code position relative to their current position. The 3-D sound beep is given using irrKlang library after finding the centre of 2-D code and its corresponding depth.

The second part of the navigation system, shown in Fig. 7, helps the blind persons orient themselves in the correct direction before starting their movement towards the desired destination. Whenever a push-button on the user's helmet is pressed, a compass sensor, integrated with the Atmel microcontroller, will send the data to the processing unit to analyse it and output to the user the current heading in degrees through the headphones using Microsoft speech library. The current heading will be continually updated with the movement of the user until the user is fully aligned with the correct direction to start moving towards the desired destination. 


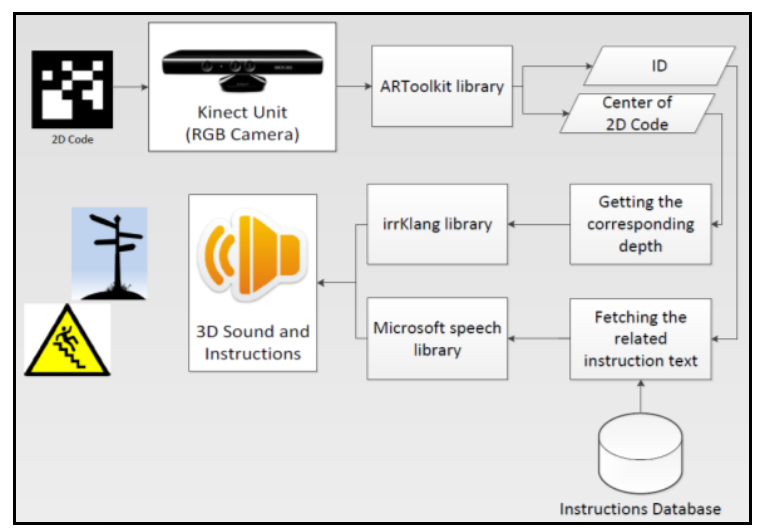

Fig. 6. Block diagram of Navigation System (part 1).

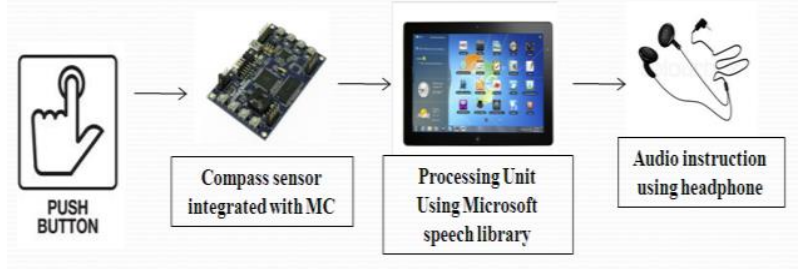

Fig. 7. Block diagram of Navigation System (part 2).

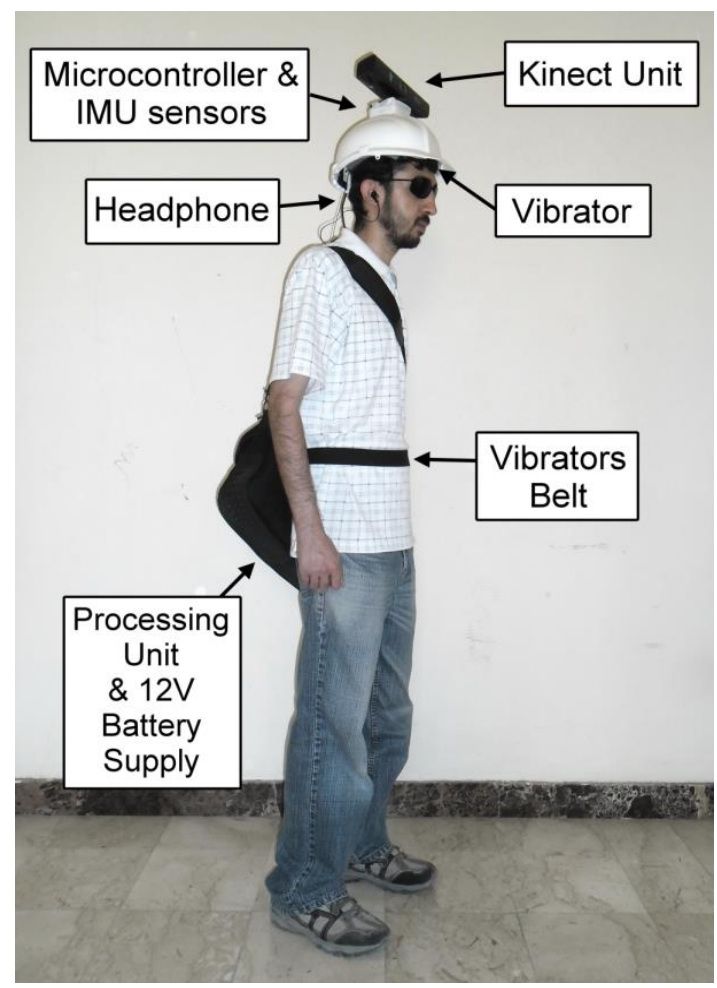

Fig. 8. Obstacle detection and guidance system.

The developed system was tested in indoor environments by both blind-folded and blind persons. Prior to starting the testing, 2-D printed codes were placed along the corridor and at a number of selected destinations. The instruction database was accordingly prepared. Also, obstacles of various sizes were placed along the path of the user. Moving obstacles were also introduced randomly while the user was heading towards the desired destination. The system identified all obstacles and assisted the user in moving with ease around the obstacles avoiding any collision with fixed or moving obstacles.

The guidance system was tested by asking the user to reach various destinations without any human help. Every time the user reached near a 2-D code, he listened to the audio instructions informing him about his current position and the next direction of movement for reaching the desired destination. After hearing the instruction, the user pressed the push-button to adjust his orientation towards the desired destination. In all cases, the user was able to reach the desired destination without any human assistance. (See Fig. 8).

\section{CONCLUSIONS}

In this paper, an indoor obstacle detection and guidance system for the blind and visually impaired has been presented. The system was designed, implemented, and successfully tested by both blind-folded and blind persons. Using four vibrators on a wearable belt and another on a light helmet, the user is gently guided to move with ease avoiding fixed or movable obstacles. It can detect obstacles within a distance of up to 4 meters in the direction of movement. By developing an algorithm to distinguish between the floor and obstacles, the system can detect even relatively small-size obstacles. The developed system also assists the user to independently reach the desired destination in unfamiliar environments by providing the user audio instructions with the help of printed 2-D codes placed at each destination (office/room/elevator) and along the corridors.

\section{ACKNOWLEDGMENT}

The authors would like to thank Emirates Association for the Blind (EAB), Sharjah, UAE, for their cooperation in testing the developed system.

\section{REFERENCES}

[1] D. Yuan and R. Manduchi, "Dynamic environment exploration using a virtual white cane," in Proc. the IEEE Computer Society Conference on Computer Vision and Pattern Recognition (CVPR '05), California, USA, June 2005, pp. 243-249.

[2] A. A. Tahat, "A wireless ranging system for the blind long-cane utilizing a smart-phone," in Proc. the 10th International Conference on Telecommunications (ConTEL '09), Croatia, June 2009, pp. 111-117.

[3] S. Shoval, I. Ulrich, and J. Borenstein, "NavBelt and the guide-cane," IEEE Robotics and Automation Magazine, vol. 10, no. 1, pp. 9-20, 2003.

[4] E. Milios, B. Kapralos, A. Kopinska, and S. Stergiopoulos, "Sonification of range information for 3-D space perception," IEEE Transactions on Neural Systems and Rehabilitation Engineering, vol. 11, no. 4, pp. 416-421, 2003.

[5] G. Sainarayanan, R. Nagarajan, and S. Yaacob, "Fuzzy image processing scheme for autonomous navigation of human blind," Applied Soft Computing Journal, vol. 7, no. 1, pp. 257-264, 2007.

[6] M. Nie, J. Ren, and Z. Li et al., "SoundView: an auditory guidance system based on environment understanding for the visually impaired people," in Proc. the 31st Annual International Conference of the IEEE Engineering in Medicine and Biology Society (EMBC '09), pp. 7240-7243, September 2009.

[7] S. Chumkamon, P. Tuvaphanthaphiphat, and P. Keeratiwintakorn, "A blind navigation system using RFID for indoor environments," in Proc. the 5th International Conference on Electrical Engineering, Electronics, Computer, Telecommunications and Information Technology (ECTI-CON '08), May 2008, pp. 765-768.

[8] D. Chen, W. Feng, Q. Zhao, M. Hu, and T. Wang, "An infrastructure-free indoor navigation system for blind people," in Intelligent Robotics and Applications, C. Y. Su, S. Rakheja, H. Liu Eds., Springer-Verlag Berlin Heidelberg, 2012, pp. 552-561.

[9] M. A. Shamsi, M. Al-Qutayri, and J. Jeedella, "Blind assistant navigation system," in Proc. 2011 Ist Middle East Conference on Biomedical Engineering (MECBME 2011), Sharjah, UAE, 2011, pp 163-166. 
[10] A. S. N. Raju, G. S. M. Chowdary, and C. Karunakar, "RF communication based assistive device for visually impaired persons," Int'l J. Research in Computer and Communication Technology, vol. 2, no. $11,2013$.

[11] R. Ivanov, "Indoor navigation system for the visually impaired," in Proc. Int'l Conference on Computer Systems and Technologies, Sofia, Bulgaria, 2010, pp. 143-149.

[12] L. A. Guerrero, F. Vasquez, and S. F. Ochoa, "An indoor navigation system for the visually impaired," Sensors, vol. 12, pp. 8236-8258, 2012.

[13] D. Dakopoulos and N. G. Bourbakis, "Wearable obstacle avoidance electronic travel aids for blind: a survey," IEEE Transactions on Systems, Man and Cybernetics, vol. 40, no. 1, pp. 25-35, 2010.

[14] Kinect-Wikipedia. [Online].

Available: http://en.wikipedia.org/wiki/Kinect

[15] F. Al-Mesaifri, M. Al-Ansari, R. Al-Shaabi, and Y. Halwani. The 3D-Kindio: A new aid to help the blind get around. Qatar University. [Online]. Available: http://www.good.is/posts/all-female-qatari-tech-team-brings-independ ent-movement-to-the-blind

[16] HCI Group. NAVI indoor navigation Kinect hack for the blind. Univ.
of
Konstanz.
[Online].
Available:

http://hci.uni-konstanz.de/blog/2011/03/15/navi/?lang=en

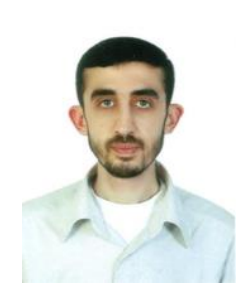

Monther Al-Shehabi received his B.Sc. degree in electrical engineering (electronics) from Ajman University of Science \& Technology in 2013. Presently, he is working in the Department of Electrical Engineering as a graduate intern.

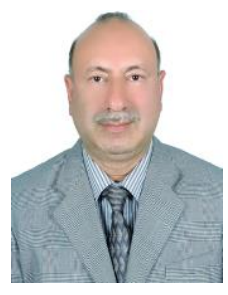

Mustahsan Mir received his B.Sc. degree in electrical engineering (with honors) from the University of Engineering \& Technology, Lahore in 1976. He received his M.S. degree in electrical engineering, M.S. degree in computer, information, and control engineering, and $\mathrm{Ph} . \mathrm{D}$. degree in electrical engineering, all from the University of Michigan, Ann Arbor, in 1979, 1981, and 1983, respectively.

Prof. Mir served in Umm Al-Qura University in Saudi Arabia from 1984 to 2001. At Umm Al-Qura University he received awards of the "Best Teacher of the College of Engineering" and the "Distinguished Teacher of the University". Presently, he is a professor and the head of the Department of Electrical Engineering at Ajman University of Science \& Technology in UAE. Prof. Mir is a member of Tau Beta Pi, Eta Kappa $\mathrm{Nu}$, and Sigma $\mathrm{Xi}$. He is a senior member of IEEE (USA) and a Fellow of IEEE (Pak)

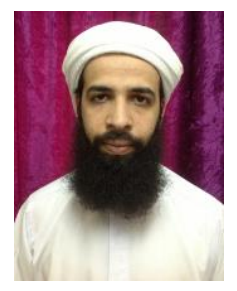

Abdullah M. Ali is a final-year student in the Department of Electrical Engineering at Ajman University of Science \& Technology, Ajman, UAE.

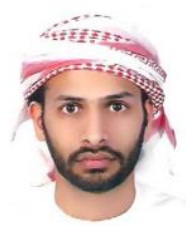

Ahmed M. Ali is a final-year student in the Department of Electrical Engineering at Ajman University of Science \& Technology, Ajman, UAE. 